\title{
Lesión del uréter tras una cirugía lumbar posterior. Caso clínico
}

\author{
C. de Quintana-Schmidt; P. Clavel-Laria y F. Bartumeus-Jené
}

Servicio de Neurocirugía. Hospital de la Santa Creu i Sant Pau. Barcelona.

\section{Resumen}

Introducción. La frecuencia de complicaciones en la cirugía lumbar por vía posterior oscila entre 1,9 y $10,8 \%$. Dentro de estas complicaciones la lesión de estructuras retroperitoneales es una complicación poco común pero potencialmente grave. La lesión del uréter es una complicación extremadamente rara existiendo en la literatura únicamente 24 casos documentados.

Caso clínico. Mujer de 43 años que durante la realización de una microdiscectomía L4-L5 se observó un sangrado profundo que fue controlado con compresión sin que existiera compromiso hemodinámico. A las 36 horas la paciente presentó un abdomen distendido y doloroso acompañado de anemia y leucocitosis. A través de un TAC abdominal y una pielografía retrógrada se diagnostico de rotura del uréter izquierdo. Se le practicó una laparotomía de urgencias y se observó una sección total del uréter izquierdo que se trató con una anastomosis termino-terminal.

Discusión. La lesión del uréter en la cirugía lumbar posterior es posible debido a la estrecha relación anatómica que presenta con el cuerpo vertebral y disco intervertebral. El nivel más frecuentemente afectado es el L4-L5. El uréter al contrario de otras estructuras retroperitoneales, se encuentra rodeado de grasa periureteral que le otorga cierta movilidad y hace más dificultosa su lesión lo que explica el bajo número de casos documentados. Aunque la sintomatología inicial puede resultar inespecífica es esencial un diagnóstico temprano para evitar complicaciones como la sepsis o incluso la pérdida del riñón. Existen varias alternativas terapéuticas, aunque el tratamiento más frecuentemente empleado es la anastomosis termino-terminal sobre todo si existe una rotura total del uréter.

PALABRAS CLAVE: Cirugía lumbar. Complicación. Discectomía. Lesión uréter. Tratamiento.

Recibido: 7-12-2009. Aceptado: 13-03-10 $\overline{\text { Ureteral injury after posterior lumbar surgery. Case }}$ report

Summary

Introduction. The percentage of complications in posterior lumbar spine surgery varies from 1,9 to $\mathbf{1 0 , 8 \%}$. Injury to retroperitoneal structures is a severe but unfrequent complication. Ureter injury is an extremely rare complication with only 24 documented cases up to date.

Case report. 43 year old woman. A deep bleeding was observed while performing a L4-L5 microdiscectomy. It was controlled with firm pressure and hemostatic agents. 36 hours later the patient developed a distended and painful abdomen associated with anemia and an increase in white blood cell count. Abdominal CT and retrograde pyelography confirmed the diagnosis of ureter injury. An emergency laparotomy was perfomed and a total section of the left ureter was treated with a end to end anastomosis.

Discussion. Ureter injury in posterior lumbar spine surgery can happen due to its close anatomical relationship with the vertebral body and disc. L4-L5 is the level most often affected. Due to the fact the ureter is surrounded by retroperitoneal fat, injury is less likely to happen and thus the low number of documented cases. Although the initial symptomatology may be inespecific, an early diagnosis is essential in order to avoid further complications such as a sepsis or a kidney loss. There are several treatment options although the most often used is the end to end anastomosis specially if there is a complete ureter injury.

KEY WORDS: Lumbar surgery. Complication. Discectomy. Ureter injury. Treatment.

Introducción

La cirugía de la hernia discal es uno de los procedimientos más frecuentes en la práctica neuroquirúrgica. 
Se calcula que en Estados Unidos se realizan 250.000 discectomías de promedio al año ${ }^{14}$. La frecuencia de complicaciones varía entre el 1,9 y $10,8 \% \%^{5,23,25}$. Dentro de estas complicaciones la lesión de estructuras retroperitoneales es una complicación poco común pero potencialmente grave.

A nivel retroperitoneal se han descrito lesiones vasculares (vasos ilíacos), vísceras y órganos abdominales ${ }^{26}$. La incidencia de lesiones a nivel retroperitoneal tras una cirugía lumbar posterior se estima entre 1:1,000 y $1: 6,000^{19}$. La lesión retroperitoneal más frecuente es la lesión de tipo vascular $^{15,16,19,26}$.

En la producción de una lesión en estructuras retroperitoneales tras una discectomía se produce frecuentemente la perforación ventral del ligamento longitudinal común vertebral anterior ${ }^{6,29}$. La incidencia de dicha perforación en la discectomía varía entre $0,016-0,06 \%$ de los $\operatorname{casos}^{26}$.

Dentro de las lesiones retroperitoneales la lesión del uréter es una complicación extremadamente rara. El primer caso fue descrito por McKay en $1954^{20}$. Desde entonces únicamente se han documentado 23 casos en la literatura $1-4,6-8,10-13,15,18,19,21,22,24,26-30$.

Presentamos el caso de una lesión a nivel del uréter tras una microdiscectomía y realizamos una revisión bibliográfica de la literatura.

\section{Caso clínico}

Mujer de 43 años con clínica de lumbalgia crónica y ciatálgia izquierda grave de años de evolución refractaria a tratamiento farmacológico. Como antecedente quirúrgico destaca microdiscectomía L4-L5 hacía 3 años. La paciente se intervino realizándose fijación transpedicular más caja intersomática en L3-L4, L4-L5 y colocación de Percudyn ${ }^{\circledR}$ en L5-S1. Durante la intervención, tras una maniobra de microdiscectomía de L4-L5 (por el lado derecho de la paciente) se observa un sangrado profundo que fue controlado con material de fibrina acompañado de compresión. No existió en ningún momento de la intervención inestabilidad hemodinámica.

La paciente presentó un post-operatorio inmediato sin complicaciones pero a las 36 horas se objetivó un abdomen distendido y doloroso. Se realizó una analítica urgente donde destacaba anemia, leucocitosis con desviación a la izquierda y PCR $>110$. Se procedió a la realización de un TAC abdominal urgente (Figura 1) donde se observó una imagen compatible con una rotura del tercio proximal del uréter izquierdo con un urinoma retroperitoneal y pélvico asociado sin presencia de hidronefrosis.

Se realizó interconsulta con el servicio de urología que realizó una pielografía retrógrada que confirmó el diagnóstico. Se practicó una laparotomía de urgencias y se observó una sección total del uréter izquierdo que se trató con una anastomosis termino-terminal. La paciente presentó un post-

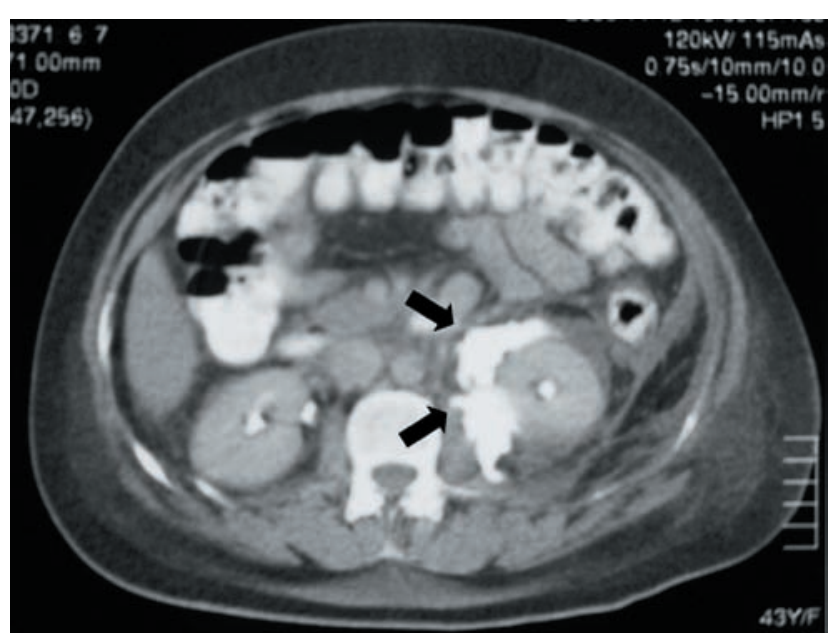

Figura 1. TAC abdominal donde se aprecia la presencia del urinoma (Flechas).

operatorio satisfactorio únicamente precisando la transfusión de dos concentrados de hematíes. Se practicó urografía de control post-quirúrgica que se muestra en la Figura 2.

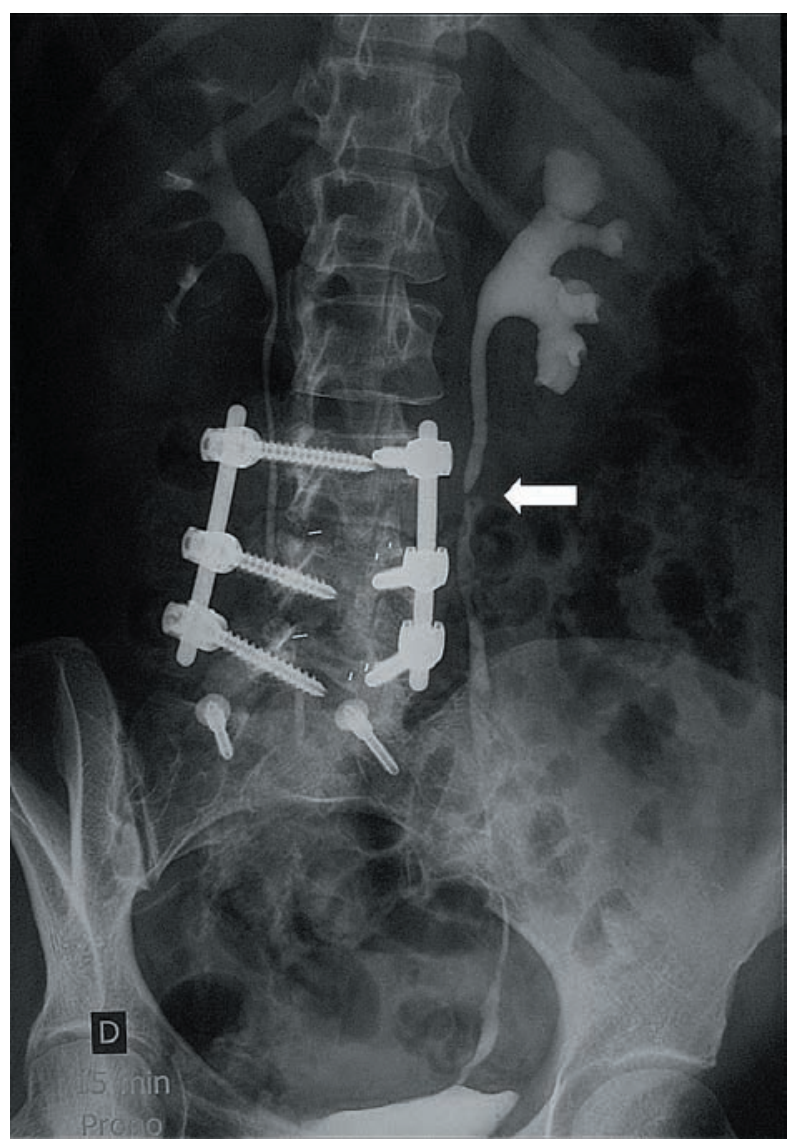

Figura 2. Urografía postquirúrgica oblicua donde se observa lesión residual del uréter (flecha) con permeabilidad del mismo y la presencia de cierta dilatación a nivel de los cálices del riñón izquierdo. 
El resultado final fue satisfactorio ya que la paciente se incorporó a la actividad laboral a los 2 meses y medio de la cirugía. Tras un año de seguimiento no presenta comorbilidades sobreañadidas.

\section{Discusión}

En la Tabla 1 se resumen los 24 casos descritos en la literatura de lesiones de uréter tras procedimientos vertebrales por vía posterior ${ }^{1-4,6-8,10-13,15,18,19-22,24,26-30}$.

La lesión del uréter en la cirugía lumbar posterior es
2011; 22: 162-166

posible debido a la estrecha relación anatómica que presenta con el cuerpo vertebral y disco intervertebral. El espacio discal más frecuentemente implicado en la lesión del uréter es el L4-L5 (16 de los 22 casos que documentan el espacio discal operado). En este espacio el uréter se encuentra lateral a los grandes vasos y entre las caras anterolaterales del cuerpo vertebral y del músculo psoas. Esto explica que lesiones de uréter vayan relacionadas en ocasiones con lesiones vasculares y viceversa ${ }^{4,6,17,19}$. En los casos descritos en la literatura en 7 de 24 casos se combinan lesiones vasculares y ureterales ${ }^{10,13,15,21,26-28}$.

Tabla 1

Resumen de los 24 casos documentados en la literatura

\begin{tabular}{|c|c|}
\hline & Número de casos (\%) \\
\hline Edad media & 39,31 [16-60] años \\
\hline \multicolumn{2}{|l|}{ Sexo } \\
\hline Mujeres & $14(58,3 \%)$ \\
\hline Hombres & $10(41,6 \%)$ \\
\hline \multicolumn{2}{|l|}{ Nivel espinal afectado } \\
\hline L4-L5 & $16(66,6 \%)$ \\
\hline L5-S1 & $4(16,6 \%)$ \\
\hline L3-L5 & $1(4,1 \%)$ \\
\hline L4-S1 & $1(4,1 \%)$ \\
\hline Desconocido & $2(8,3 \%)$ \\
\hline \multicolumn{2}{|l|}{ Síntomas más frecuentes } \\
\hline Dolor abdominal & $20(83,3 \%)$ \\
\hline Fiebre & $15(62,5 \%)$ \\
\hline Íleo & $4(16,6 \%)$ \\
\hline Leucocitosis & $4(16,6 \%)$ \\
\hline Shock séptico & $3(12,5 \%)$ \\
\hline Tiempo medio del diagnóstico tras la cirugía & 17,7 días [Mismo acto quirúrgico - 60 días] \\
\hline \multicolumn{2}{|l|}{ Afectación vascular } \\
\hline $\mathrm{Si}$ & $7(29,1 \%)$ \\
\hline No & $17(70,8 \%)$ \\
\hline \multicolumn{2}{|l|}{ Tratamiento realizado } \\
\hline Anastomosis termino-terminal & $10(41,6 \%)$ \\
\hline Stent & $6(25 \%)$ \\
\hline Nefrectomía & $4(16,6 \%)$ \\
\hline Autotransplante & $2(8,3 \%)$ \\
\hline nastomosis urétero-ileal & $1(4,1 \%)$ \\
\hline Nefroureterostomía & $1(4,1 \%)$ \\
\hline
\end{tabular}


La lesión vascular es más frecuente que la lesión ureteral a nivel retroperitoneal debido a que los grandes vasos están relativamente fijados intraabdominalmente en comparación al uréter que normalmente esta envuelto de grasa periureteral que le otorga cierta adaptabilidad frente a las lesiones $^{10,15,17,19,22}$.

La relación anatómica entre el uréter y el cuerpo vertebral se estrecha aún más durante la cirugía lumbar posterior ya que el paciente es colocado en decúbito prono. Esto provoca un incremento de la presión intrapélvica que presiona el uréter contra el cuerpo vertebral y el espacio discal aumentando la probabilidad de lesión del mismo ${ }^{6,17,19}$.

Se han descrito ciertos factores que predisponen a los pacientes ha sufrir lesiones a nivel ureteral: pacientes delgados, defectos $\mathrm{y} / \mathrm{o}$ ausencia de la parte anterior del annulus del disco, cicatrices o adhesiones retroperitoneales tras una cirugía o proceso inflamatorio previo e incorrecta colocación del paciente ${ }^{6,9,17,19}$. Creemos que la cirugía previa que se realizó en el espacio L4-L5 actuó como factor predisponerte.

Existen dos formas de lesionar el uréter a través de una cirugía lumbar posterior. La más frecuente es tras una perforación de la parte lateral del ligamento longitudinal anterior mientras se realiza la discectomía ${ }^{4,6,29}$. Este mecanismo ha sido demostrado experimentalmente en cadáveres ${ }^{19}$. Característicamente se lesiona el uréter contralateral al lado donde se realiza el procedimiento debido al paso tangencial de los instrumentos al realizar la discectomia ${ }^{4,19,29}$. La otra forma de lesionar el uréter es si se produce una violación del espacio intertransverso lo que puede provocar la lesión del uréter ipsilateral al lado del procedimiento ${ }^{4}$. Cuando se lesiona el uréter se pueden producir dos tipos de secciones; la parcial y la completa. El tipo de sección más frecuente es la parcial $^{1,10}$.

Habitualmente existe una demora en el diagnóstico de una lesión a nivel del uréter tras una cirugía lumbar posterior. Tras la revisión bibliográfica se observa que el tiempo medio hasta el diagnóstico de la lesión es de 17,7 días con un intervalo entre en el mismo acto quirúrgico y 60 días. Este retraso en el diagnóstico se debe principalmente a dos causas. En primer lugar, es debido a que en la mayoría de los casos los síntomas iniciales son inespecíficos (fiebre, dolor abdominal, íleo, leucocitosis.... $)^{1,4,11,19,29}$. En segundo lugar, los cirujanos no contemplan esta complicación bien por desconocimiento o bien por tratarse de una complicación extremadamente rara. Es esencial un diagnóstico temprano de este tipo de lesiones ya que si el diagnóstico es precoz la mayoría de los casos tiene buen pronóstico. Sin embargo, si existe una demora excesiva el pronóstico empeora y se puede producir la pérdida del riñón y/o un cuadro séptico ${ }^{13,15,22,24,27}$. En los cuatro casos de nefrectomía existió una demora de más de 17 días. No se ha descrito ningún caso de muerte como consecuencia de esta compli-
2011; 22: 162-166

cación.

Debido a los pocos casos documentados en la literatura no existe consenso en cuál es el tratamiento de elección. El tratamiento más frecuentemente empleado es la anastomosis termino-terminal y es el más aconsejable cuando existe una rotura total del uréter $^{2-4,6,7,13,19,20,28,30}$. Si la rotura es parcial algunos autores aconsejan la colocación de un stent ${ }^{1,8,10,15,18,21}$. Otras alternativas son el autotransplante renal si existe excesiva tensión a nivel de la anastomosis, la anastomosis urétero-ileal, la nefroureterostomía y en los casos más graves la nefrectomía ${ }^{11-13,22,24,26,27,29,30}$.

\section{Conclusión}

Aunque poco frecuente la lesión del uréter tras una cirugía lumbar posterior debe tenerse en cuenta en el diagnóstico diferencial de las posibles complicaciones post-quirúrgicas. Un diagnóstico precoz es esencial para el buen pronóstico de los pacientes mientras que una demora excesiva puede llevar a un cuadro séptico o incluso a la pérdida del riñón.

\section{Bibliografía}

1. Altebarmakian, V.K, Davis, R.S., Khuri, F.J.: Ureteral injury associated with lumbar disk surgery. Urology. 1981; 17: 462-464.

2. Bec, A.: Ureteric injury during laminectomy for a prolapsed disc. Br J Urol. 1989; 63: 552-553.

3. Borski, A., Smith, R.: Ureteral injury in lumbar disk operation. J. Neurosurg. 1960; 17: 925-928.

4. Cho, K.T., Im, S.H., Hong, S.K.: Ureteral injury after inadvertent violation of the intertransverse space during posterior lumbar diskectomy: a case report. Surg Neurol. 2008 ;69: 135-137.

5. Davis, R.A.: A long-term outcome analysis of 984 surgically treated herniated lumbar discs. J Neurosurg. 1994; 80: 415-421.

6. Defay, P., L'Hermite, J., Wiederkehr, P., Raul, P., Guillemin, P.: An unusual ureteric injury. Br J Urol. 1986; $58: 567$.

7. Demirkesen, O., Tunc, B., Ozkan, B.: A rare complication of lumbar disk surgery: ureteral avulsion. Int Urol Nephrol. 2006; 38: 459-461.

8. Flam, T.A., Spitzenpfeil, E., Zerbib, M., Steg, A., Debre, B.: Complete ureteral transection associated with percutaneous lumbar disk nucleotomy. J Urol. 1992; 148: 1249-1250.

9. Fruhwirth, J., Koch, G., Amann, W., Hauser, H., Flaschka, G.: Vascular complications of lumbar disc surgery. Acta Neurochir (Wien). 1996; 138: 912-916.

10. Gangai, M.P.: Ureteral injury incident to lumbar disc surgery. Case report. J Neurosurg. 1972; 36: 90-92.

11. Gayer, G., Caspi, I., Garniek, A., Hertz, M., Apter, S.: Perirectal urinoma from ureteral injury incurred during spinal 
surgery mimicking rectal perforation on computed tomography scan. Spine. 2002; 27: E451-453.

12. Hekal, I.A., Mohsen, T., Nabeeh, A.: Ureteric injury after lumbosacral discectomy: a case report and review of the literature. J Trauma. 2008; 64: 1387-1391.

13. Heller, V., Osterhage, H.R., Heckl, W., Frohmüller, H.: Ureteral injuries in intervertebral lumbar disk surgery. Urologe A. 1986; 25: 347-350.

14. Hernández-Pérez, P.A., Prinzo-Yamurri, H.: Analysis of the lumbar discectomy complications. Neurocirugía 2005; 16: 419-426.

15. Holscher, E.C.: Vascular and visceral injuries during lumbar-disc surgery. J Bone Joint Surg Am. 1968; 50: 383393.

16. Inamasu, J., Guiot, B.H.: Vascular injury and complication in neurosurgical spine surgery. Acta Neurochir (Wien). 2006; 148: 375-387.

17. Isiklar, Z.U., Lindsey, R.W., Coburn, M.: Ureteral injury after anterior lumbar interbody fusion. A case report. Spine. 1996; 21: 2379-2382.

18. Kern, H.B., Barnes, W., Malament, M.: Lumbar laminectomy and associated ureteral injury. J Urol. 1969; 102: 675-677.

19. Krone, A., Heller, V., Osterhage, H.R.: Ureteral injury in lumbar disc surgery. Acta Neurochir (Wien). 1985; 78: 108112.

20. McKay, H.W., Haird, H.H., Justis, H.R.: Management of ureteral injuries. J Am Med Assoc. 1954; 154: 202-205.

21. Moore, C.A., Cohen, A.: Combined arterial, venous, and ureteral injury complicating lumbar disk surgery. Am J Surg. 1968; 115: 574-577.

22. Noyes, D.T., Morrisseau, P.M.: Ureteral transection secondary to lumbar disk surgery. Urology. 1982; 19: 651652.

23. Pappas, C.T., Harrington, T., Sonntag, V.K.: Outcome analysis in 654 surgically treated lumbar disc herniations. Neurosurgery. 1992; 30: 862-866.

- 24- Parker, J.M.: Ureteral injury secondary to lumbar disk operation. J Urol. 1971; 105: 85.

25. Ramirez, L.F., Thisted, R.: Complications and demographic characteristics of patients undergoing lumbar discectomy in community hospitals. Neurosurgery. 1989; 25: 226-230.

26. Ríos González, E., Ramón de Fata Chillón, F., Tabernero Gómez, A., Núñez Mora, C., Hidalgo Togores, L., de la Peña Barthel, J.J.: Iatrogenic injury of the lumbar ureter and iliac vessels after lumbar discectomy: urologic treatment using kidney autotransplantation. Actas Urol Esp. 2002; 26: 504-508.

27. Sandoz, I., Hodges, C.V.: Ureteral injury incident to lumbar disk operation. J Urol. 1965; 93: 687-689.

28. Tainio, H., Kylmala, T.: Rupture of the ureter: an unexpected complication of microdiskectomy. BJU Int. 1999; 84: 369-370.

29. Trinchieri, A., Montanari, E., Salvini, P., Berardinelli, L., Pisani, E.: Renal autotransplantation for complete ureteral avulsion following lumbar disk surgery. J Urol. 2001; 165: 1210-1211.

30. Zinman, L.M., Libertino, J.A., Roth, R.A.: Management of operative ureteral injury. Urology. 1978; 12: 290303.

De Quintana-Schmidt, C.; Clavel-Laria, P.; BartumeusJené, F.: Lesión del uréter tras una cirugía lumbar posterior. Caso clínico. Neurocirugía 2011; 22: 162-166.

Correspondencia: Hospital de la Santa Creu i Sant Pau, Departamento de Neurocirugía, C $\backslash$ Sant Antoni Maria Claret 16708025 Barcelona.

Correo electrónico: cquintana@santpau.es /cqs_7@hotmail.com. 